\section{Transtornos mentais em comunidade atendida pelo Programa Saúde da Família}

\author{
Mental disorders in a community assisted by the \\ Family Health Program
}

\author{
${ }^{1}$ Laboratório de Psiquiatria \\ Molecular, Hospital de \\ Clínicas de Porto Alegre, \\ Porto Alegre, Brasil. \\ 2 Departamento de \\ Psiquiatria e Medicina Legal, \\ Universidade Federal do \\ Rio Grande do Sul, \\ Porto Alegre, Brasil. \\ Correspondência \\ D. M. Gonçalves \\ Laboratório de Psiquiatria \\ Molecular, Hospital de \\ Clínicas de Porto Alegre. \\ Rua 28 de outubro 662, \\ Santa Cruz do Sul, RS \\ 96815-710, Brasil. \\ danielmgpoa@yahoo.com.br
}

\begin{abstract}
The first objective of this study was to estimate the prevalence of mood, anxiety, and somatoform disorders (MASD) using the screening tool Self-Reporting Questionnaire-20 Items (SRQ-20) in a community assisted by the Family Health Program (FHP) in Santa Cruz do Sul, Rio Grande do Sul, Brazil. The second goal was to verify associations between demographic variables and MASD. We invited all residents $\geq$ 14 years of age to participate. The total sample consisted of 1,122 subjects. MASD prevalence was 38\% (95\%CI: 35.12-40.88). Female gender, low schooling, and unemployment were independently associated with MASD. The high prevalence of MASD observed in this study confirms the relevance of these disorders for public health planning, and could also help explain the high suicide rate in Santa Cruz do Sul in the last decade (4.66 times the national rate). Finally, the study highlights the importance of including mental health in the FHP priority agenda in Brazil.
\end{abstract}

Mental Disorders; Mental Health; Family Health Program
Daniel Maffasioli Gonçalves 1

Flavio Kapczinski 1,2

\section{Introdução}

Estudos epidemiológicos têm demonstrado prevalências de transtornos mentais ao longo da vida entre $12,2 \%$ e 48,6\%, dependendo da população estudada e das características do instrumento de rastreamento ou diagnóstico utilizadas. Aproximadamente $90 \%$ dos casos de transtornos mentais na população geral são transtornos de humor, ansiedade e/ou somatoformes (THAS) 1,2 Esses transtornos são agrupados muitas vezes sob diferentes denominações devido ao fato de compartilharem grande parte de sintomas. Morbidade psiquiátrica menor, distúrbios psiquiátricos menores e transtornos mentais comuns são os mais utilizados 2,3,4,5,6, embora estes termos também sejam usados para designar uma síndrome descrita por Goldberg \& Huxley 7 , caracterizada pelos sintomas de insônia, fadiga, irritabilidade, esquecimento, dificuldade de concentração e queixas somáticas.

Transtornos mentais em geral causam considerável impacto em termos de morbidade, prejuízos na funcionalidade e diminuição da qualidade de vida de seus portadores. O estudo Global Burden of Disease, conduzido pela Organização Mundial da Saúde (OMS) 1 durante a década de 1990, estimou o impacto das 130 condições médicas mais prevalentes no mundo em termos de morbidade e mortalidade analisando dados oriundos de 54 países. Os resultados demonstraram que da totalidade dos anos vividos com inca- 
pacidade no mundo devido a doenças, $30,8 \%$ são decorrentes de transtornos mentais. O mesmo estudo mostra que dentre as dez primeiras causas de anos vividos com incapacidade no mundo sete são transtornos mentais 1 .

Visto a alta prevalência de transtornos mentais e o impacto considerável na vida dos indivíduos afetados, tem-se sugerido a necessidade de implementação de políticas públicas na área. Para tanto é necessário dispor de informações adequadas a fim de realizar-se uma análise objetiva das situações sobre as quais se pretende atuar. O uso racional dos recursos disponíveis torna imprescindível a busca permanente de instrumentos que auxiliem na tomada de decisões, sendo que os indicadores de saúde cumprem este papel 8,9.

Os estudos epidemiológicos em saúde mental propiciam compreender mais claramente a freqüência com que esses transtornos ocorrem na população em geral, avaliar suas conseqüências diretas e indiretas em nível individual, familiar e social, além de trazer à tona suas associações com variáveis sócio-demográficas a fim de orientar o reconhecimento de grupos de risco ${ }^{8}$. Deve ser prioritariamente realizado em bases populacionais, pois estas representam de forma mais fidedigna a realidade. Entretanto, esse tipo de estudo no Brasil ainda é escasso, principalmente quando consideramos tratar-se de um país de dimensões continentais. Um dos motivos para essa escassez é a necessidade de aportes consideráveis de recursos financeiros para a sua realização ${ }^{9}$.

O Self-Reporting Questionnaire - 20 Itens (SRQ-20) é um instrumento de rastreamento de THAS auto-respondido, de fácil compreensão, rápida execução e muito barato, atualmente recomendado pela OMS para uso na clínica e pesquisa 10 . Vários países de culturas diferentes têm utilizado amplamente esse instrumento 11 . No Brasil, o SRQ-20 foi validado pela primeira vez na década de 1980 comparando-o com o Clinical Interview Schedule (CIS) 12, baseado nos critérios diagnósticos do Diagnostic and Statistical Manual of Mental Disorders (DSM-III) 13, quando estabeleceu-se como ponto de corte $7 / 8$ para mulheres e $5 / 6$ para homens, com sensibilidade de $83 \%$ e especificidade de $80 \% 2$.

Recentemente, conduzimos um estudo de atualização do desempenho do SRQ-20 como instrumento de rastreamento de THAS comparando-o com entrevista psiquiátrica estruturada utilizando o Structured Clinical Interview for DSM Disorders (SCID) 14, baseado na versão corrente do DSM-IV-TR 15. Obtivemos como ponto de corte ótimo 7/8 para toda amostra, mesmo ponto de corte para homens e mulheres quando analisados em separado. Nesse ponto de corte a sensibilidade é de $86,33 \%$ e a especificidade de $89,31 \% 16$

O objetivo deste estudo é verificar a prevalência de casos suspeitos de THAS em uma comunidade atendida pelo Programa Saúde da Família (PSF) em Santa Cruz do Sul, Rio Grande do Sul, Brasil, utilizando-se o instrumento de rastreamento psiquiátrico SRQ-20, e verificar possíveis associações entre presença suspeita de THAS e variáveis demográficas.

\section{Métodos}

\section{Amostra}

O estudo foi conduzido em uma área delimitada de Santa Cruz do Sul, atendida por uma equipe de PSF, sendo acompanhada por médico, profissionais de enfermagem e quatro agentes comunitários de saúde. Cada agente é responsável por aproximadamente um quarto do total de moradores. Na região residem 2.759 pessoas, sendo 1.700 maiores de 14 anos.

Foram considerados critérios de inclusão: indivíduos maiores de 14 anos, moradores da área e que aceitaram participar voluntariamente da pesquisa. Utilizou-se como critério de exclusão apenas a presença de comprometimento mental que impossibilitasse a participação, basicamente retardo mental e demência de moderada a grave. No total, $1.228(72,23 \%)$ indivíduos participaram do estudo, sendo que 106 foram eliminados devido a questionários incompletos, resultando em 1.122 pessoas que foram consideradas na análise, o que corresponde a $66 \%$ da população da área maior de 14 anos.

\section{Delineamento}

Trata-se de um estudo transversal quantitativo realizado no período de 10 de fevereiro a 10 de dezembro de 2006. O trabalho constituiu-se em aplicação de questionário sobre dados demográficos e SRQ-20 pelas quatro agentes comunitárias de saúde responsáveis pela área, que participaram do estudo como aplicadoras de forma voluntária. Nas visitas domiciliares regulares realizadas pelas agentes durante o período de estudo todos os moradores acima de 14 anos da área eram convidados a participar. Quando um morador não estava presente na residência durante a visita, o convite para participar da pesquisa era feito verbalmente por intermédio do familiar presente e depois por escrito, sendo, quando necessário, agendado um horário com a agente comunitária de saúde para a participação na pesquisa. $\mathrm{O}$ convite em cada residência foi feito mais de uma 
vez, visto que a média de visitas domiciliares por agentes comunitários de saúde neste PSF é de aproximadamente 0,8 visita por domicílio ao mês. Os questionários foram respondidos individualmente pelos participantes. No caso de o entrevistado ser analfabeto, os instrumentos eram lidos por um terceiro, preferencialmente a agente comunitária de saúde. Isso porque o SRQ-20 objetiva incluir todo o tipo de paciente, inclusive os analfabetos 10 .

\section{Instrumentos}

\section{- Questionário sobre dados demográficos}

Trata-se de um questionário auto-respondido composto por oito perguntas acerca de dados demográficos e de identificação. São elas: sexo, idade, etnia, nível de escolaridade, situação conjugal e ocupação. Na variável situação conjugal considerou-se como casamento toda união oficial e união não-oficial com um ano ou mais de duração.

\section{- SRQ-20}

O SRQ-20 é a versão de vinte itens oriunda do SRQ-24 utilizada para rastreamento de THAS 10. As respostas são do tipo sim/não. Cada resposta afirmativa pontua com o valor 1 para compor o escore final por meio do somatório destes valores. Os escores obtidos estão relacionados com a probabilidade de presença de THAS, sendo que variam de 0 (nenhuma) a 20 (extrema probabilidade).

O ponto de corte que utilizamos foi $7 / 8$ para toda a amostra, com sensibilidade de $86,33 \%$ e especificidade de 89,31\% para detecção de THAS, conforme citado na Introdução 16.

\section{Preparação e suporte aos aplicadores}

As quatro agentes comunitárias de saúde foram treinadas em dez encontros de três horas cada, para sensibilização e capacitação em saúde mental, sendo cinco encontros antes do início do estudo e cinco no decorrer do estudo. Além desses, participaram de encontros quinzenais realizados pelo primeiro autor a fim de receberem suporte técnico e emocional.

\section{Análise estatística}

Os dados foram compilados no programa SPSS versão 12.0 (SPSS Inc., Chicago, Estados Unidos) com dupla digitação, posterior validate e limpeza do banco de dados. As análises foram realizadas no mesmo programa. Foram considerados casos suspeitos de THAS os que pontuaram no SRQ-20 com escore final de 8 ou mais, originando a variável dependente dicotômica presença ou ausência de suspeita de THAS.

As médias aritméticas e desvios-padrão (DP) foram calculados para os dados contínuos, e freqüências foram calculadas para as variáveis qualitativas. Realizou-se análise descritiva da população segundo as variáveis sócio-demográficas em estudo (sexo, idade, etnia, situação conjugal, escolaridade e situação ocupacional). A primeira análise para verificar associação de variáveis sócio-demográficas com risco de THAS foi univariada. Foram calculadas as odds ratios (OR), e a significância estatística foi verificada usando-se o teste do qui-quadrado. Após essa primeira análise, realizou-se regressão logística para controle das variáveis de confusão utilizando-se as variáveis que apresentaram associação significativa na análise univariada. A regressão logística foi utilizada para analisar a independência da associação de cada variável demográfica que mostrou-se associada com THAS na análise univariada. No modelo final foram incluídas apenas as variáveis associadas de forma independente. Foram calculadas as OR ajustadas para as variáveis que apresentaram associação significativa com THAS na análise multivariada, sendo a significância estatística verificada pelo teste do qui-quadrado. Consideramos valor de $\mathrm{p}<0,05 \mathrm{e}$ intervalo de 95\% de confiança (IC95\%).

\section{Aspectos éticos}

Este tipo de estudo apresenta risco mínimo de acordo com as Normas de Pesquisa em Saúde do Conselho Nacional de Saúde. O protocolo do estudo foi submetido ao Comitê de Ética em Pesquisa do Hospital de Clínicas de Porto Alegre e à análise da Secretaria Municipal de Saúde do Município de Santa Cruz do Sul. Todos os participantes preencheram um Termo de Consentimento Livre e Esclarecido.

\section{Resultados}

Dos 1.122 indivíduos que participaram do estudo, 693 (61,8\%) eram do sexo feminino e a idade média geral foi de 35,46 (DP = 15,27). A maioria foi de brancos $(58,4 \%)$, casados $(57,5 \%)$ e com Ensino Fundamental incompleto $(60,3 \%)$, o que corresponde ao primeiro grau incompleto no antigo sistema de classificação. Em relação à situação ocupacional, 561 (50\%) estavam trabalhando no momento das entrevistas e os demais se distribuíram nas outras categorias consideradas, que são: estudantes (13,5\%), desempregados (13,8\%), 
em benefício-saúde ou aposentados por invalidez (10,6\%), aposentados por tempo de serviço $(2,3 \%)$ e donas-de-casa $(9,7 \%)$. As características da amostra estão descritas na Tabela 1.

A média de escores finais no SRQ-20 foi de $6,5(\mathrm{DP}=4,79)$, com mediana 6 (quartil - Q25 = 3 ; Q75 = 10). Utilizando-se como ponto de corte 7/8, 426 indivíduos foram considerados casos suspeitos de THAS, representando uma prevalência de 38\% (IC95\%: 35,12-40,88).

Na Tabela 1 estão apresentadas as prevalências de THAS distribuídas de acordo com as va- riáveis demográficas consideradas, e na Tabela 2 os valores de OR resultantes da primeira análise para verificação de associações. Inicialmente, cada uma das variáveis consideradas foi analisada em separado (análise univariada). Houve associação estatisticamente significativa para sexo $\left(\chi^{2}=41,486 ; \mathrm{p}<0,001\right)$, idade $\left(\chi^{2}=15,672\right.$; $\mathrm{p}=0,003)$, situação conjugal $\left(\chi^{2}=18,971 ; \mathrm{p}<\right.$ $0,001)$, escolaridade $\left(\chi^{2}=20,291 ; \mathrm{p} ;<; 0,001\right)$ e ocupação $\left(\chi^{2}=60,673 ; \mathrm{p}<0,001\right)$. Ou seja, apenas etnia não apresentou associação $\left(\chi^{2}=1,878\right.$; $\mathrm{p}=0,391)$.

Tabela 1

Características da amostra e prevalência de casos suspeitos de transtornos de humor, ansiedade e somatização (THAS) * distribuídos por variáveis demográficas.

\begin{tabular}{|c|c|c|c|c|}
\hline \multirow[t]{2}{*}{ Variável } & \multicolumn{2}{|c|}{$\begin{array}{c}\text { Total } \\
(n=1.122 ; 100,0 \%)\end{array}$} & \multicolumn{2}{|c|}{$\begin{array}{c}\text { THAS } \\
(n=426 ; 38,0 \%)\end{array}$} \\
\hline & $\mathrm{n}$ & $\%$ & $\mathrm{n}$ & $\%$ \\
\hline \multicolumn{5}{|l|}{ Sexo } \\
\hline Masculino & 429 & 38,2 & 112 & 26,1 \\
\hline Feminino & 693 & 61,8 & 314 & 45,3 \\
\hline \multicolumn{5}{|l|}{ Idade (anos) } \\
\hline $14-24$ & 340 & 30,3 & 104 & 30,6 \\
\hline $25-34$ & 236 & 21,0 & 85 & 36,0 \\
\hline $35-44$ & 247 & 22,0 & 108 & 43,7 \\
\hline $45-54$ & 162 & 14,4 & 73 & 45,1 \\
\hline$>54$ & 137 & 12,2 & 56 & 40,9 \\
\hline \multicolumn{5}{|l|}{ Etnia } \\
\hline Negro & 198 & 17,6 & 67 & 33,8 \\
\hline Branco & 655 & 58,4 & 252 & 38,5 \\
\hline Pardo & 269 & 24,0 & 107 & 39,8 \\
\hline \multicolumn{5}{|l|}{ Situação conjugal } \\
\hline Casados ** & 645 & 57,5 & 243 & 37,7 \\
\hline Solteiros & 327 & 29,1 & 105 & 32,1 \\
\hline Divorciados & 87 & 7,8 & 49 & 56,3 \\
\hline Viúvos & 63 & 5,6 & 29 & 46,0 \\
\hline \multicolumn{5}{|l|}{ Escolaridade } \\
\hline Analfabetos & 96 & 8,6 & 44 & 45,8 \\
\hline 1-4 anos do Ensino Fundamental & 677 & 60,3 & 275 & 40,6 \\
\hline 5-8 anos do Ensino Fundamental & 161 & 14,3 & 62 & 38,5 \\
\hline Ensino Médio incompleto & 103 & 9,2 & 25 & 24,3 \\
\hline Ensino Médio completo & 85 & 7,6 & 20 & 23,5 \\
\hline \multicolumn{5}{|l|}{ Ocupação } \\
\hline Trabalhadores em atividade & 561 & 50,0 & 177 & 31,6 \\
\hline Estudantes & 152 & 13,5 & 37 & 24,3 \\
\hline Desempregados & 155 & 13,8 & 81 & 52,3 \\
\hline \multicolumn{5}{|l|}{ Em benefício-saúde/ } \\
\hline aposentados por invalidez & 119 & 10,6 & 66 & 55,0 \\
\hline Aposentados por tempo de serviço & 26 & 2,3 & 8 & 30,8 \\
\hline Donas-de-casa & 109 & 9,7 & 57 & 52,3 \\
\hline
\end{tabular}

* Caso suspeito de THAS: considerado quando o escore final no Self-Reporting Questionnaire-20 Itens (SRQ-20) for igual ou maior que 8 ;

** Considerados casados todos com casamento formalizado (civil ou religioso) ou união estável de mais de um ano. 
Análise univariada; apresentação de odds ratios (OR) não-ajustadas para verificar associação entre presença suspeita de transtornos de humor, ansiedade e somatização (THAS) * e variáveis demográficas, com intervalos de 95\% de confiança (IC95\%) e valor de $\mathrm{p}$

\begin{tabular}{|c|c|c|c|}
\hline Variável & OR & IC95\% & $p$ \\
\hline \multicolumn{4}{|l|}{ Sexo } \\
\hline Masculino & 1,00 & & \\
\hline Feminino & 2,34 & $1,80-3,04$ & $<0,001$ \\
\hline \multicolumn{4}{|l|}{ Idade (anos) } \\
\hline $14-24$ & 1,00 & & \\
\hline $25-34$ & 1,28 & $0,90-1,82$ & 0,173 \\
\hline $35-44$ & 1,76 & $1,25-2,48$ & 0,001 \\
\hline $45-54$ & 1,86 & $1,26-2,74$ & 0,002 \\
\hline$>54$ & 1,57 & $1,04-2,37$ & 0,032 \\
\hline \multicolumn{4}{|l|}{ Etnia } \\
\hline Negro & 1,00 & & \\
\hline Branco & 1,22 & $0,88-1,71$ & 0,238 \\
\hline Pardo & 1,29 & $0,88-1,89$ & 0,190 \\
\hline \multicolumn{4}{|l|}{ Situação conjugal } \\
\hline Casados ** & 1,00 & & \\
\hline Solteiros & 0,78 & $0,59-1,04$ & 0,088 \\
\hline Divorciados & 2,13 & $1,36-3,35$ & 0,001 \\
\hline Viúvos & 1,41 & $0,84-2,37$ & 0,195 \\
\hline \multicolumn{4}{|l|}{ Escolaridade } \\
\hline Analfabetos & 2,75 & $1,45-5,23$ & 0,002 \\
\hline 1-4 anos do Ensino Fundamental incompleto & 2,22 & $1,32-3,75$ & 0,003 \\
\hline 5-8 anos do Ensino Fundamental completo & 2,03 & $1,12-3,68$ & 0,019 \\
\hline Ensino Médio incompleto & 1,04 & $0,53-2,04$ & 0,905 \\
\hline Ensino Médio completo & 1,00 & & \\
\hline \multicolumn{4}{|l|}{ Ocupação } \\
\hline Trabalhadores em atividade & 1,00 & & \\
\hline Estudantes & 0,70 & $0,46-1,05$ & 0,086 \\
\hline Desempregados & 2,37 & $1,65-3,41$ & $<0,001$ \\
\hline Aposentados por invalidez/em benefício-saúde & 2,70 & $1,81-4,04$ & $<0,001$ \\
\hline Aposentados por tempo de serviço & 0,96 & $0,41-2,26$ & 0,933 \\
\hline Donas-de-casa & 2,38 & $1,57-3,60$ & $<0,001$ \\
\hline
\end{tabular}

* Caso suspeito de THAS: considerado quando o escore final no Self-Reporting Questionnaire-20 Itens (SRQ-20) for igual ou maior que 8;

** Considerados casados todos com casamento formalizado (civil ou religioso) ou união estável de mais de um ano.

Mulheres apresentaram maior chance de apresentar THAS. Houve uma correlação linear positiva entre idade e THAS até a categoria 54 anos ou mais, quando verificou-se um decréscimo na prevalência e conseqüentemente dos valores de OR. Em relação à situação conjugal, tomando como referência os casados, as categorias solteiros e viúvos não apresentaram diferença significativa, havendo no entanto diferença em relação aos divorciados (maior chance de THAS). Em relação à escolaridade, percebe-se uma correlação linear inversa entre chances de apresentar THAS e nível de escolaridade, sendo que tomando como referência a categoria ensino médio completo, houve diferenças significativas com todas as outras categorias inferiores, exceto ensino médio incompleto. Por fim, para a variável ocupação, tomando-se como referência a categoria trabalhadores em atividade, houve maiores chances de as categorias desempregado, aposentado por invalidez ou em benefício-saúde e dona-de-casa apresentarem THAS, enquanto que não houve diferença em relação a estudantes e aposentados por tempo de serviço. 
Na primeira etapa da análise multivariada foram incluídas todas as variáveis que apresentaram associação significativa com THAS na análise univariada, ou seja, sexo, idade, situação conjugal, escolaridade e ocupação. Idade e situação conjugal perderam significância quando ajustadas por sexo, escolaridade e ocupação. Dessa forma foram desconsideradas nas etapas seguintes e no modelo final. Observou-se efeito independente das variáveis sexo, escolaridade e ocupação, nesta ordem de importância.

Na Tabela 3 estão apresentadas as OR ajustadas de cada variável para as demais variáveis que apresentaram associação independente com THAS na análise multivariada. Sexo feminino manteve-se associado $(\mathrm{p}<0,001)$. Para a variável escolaridade, houve uma relação inversa entre chance de apresentar THAS e nível de escolaridade. Tomando por referência a categoria ensino médio completo, observaram-se OR significativamente maiores entre os indivíduos com Ensino Fundamental e analfabetos. Por fim, desempregados, pessoas com benefício por problemas de saúde ou aposentados por invalidez e donas-decasa apresentaram OR significativamente maiores quando comparados com trabalhadores em atividade.

\section{Discussão}

Os principais achados deste estudo, realizado em uma população de baixo nível sócio-econômico atendida por PSF em Santa Cruz do Sul, foram a alta prevalência de 38\% (IC95\%: 35,12-40,88) de THAS e a associação destes transtornos com sexo feminino, baixa escolaridade e situação ocupacional desfavorável. Considerando-se a sensibilidade $(86,33 \%)$ e a especificidade $(89,31 \%)$ do instrumento utilizado, pode-se estimar a prevalência corrigida em 36\% (IC95\%: 33,19-38,89; cálculo: $\mathrm{Pc}=\mathrm{Po}+\mathrm{E}-1 / \mathrm{S}+\mathrm{E}-1$, em que $\mathrm{Pc}=$ prevalência corrigida; Pó = prevalência observada; $\mathrm{E}$ = especificidade; $\mathrm{S}=$ sensibilidade) ${ }^{8}$. A alta taxa de participação (66\%) dos moradores da área onde a pesquisa foi conduzida é um fator que fortalece os resultados do estudo.

Em revisão da literatura, encontramos quatro estudos epidemiológicos de base populacional utilizando o SRQ-20 no Brasil. Um desses estudos foi realizado durante o ano de $2001 \mathrm{em}$ quatro áreas de baixo nível sócio-econômico da zona urbana do Município de São Paulo, sendo duas cobertas pelo PSF. As características deste estudo são muito semelhantes à nossa: amostra de indivíduos com 14 anos ou mais, de uma tornos de humor, ansiedade e somatização (THAS) ** e variáveis demográficas, com intervalos de 95\% de confiança (IC95\%) e valor de $\mathrm{p}$

\begin{tabular}{|c|c|c|c|}
\hline Variável & OR ajustadas & IC95\% & $\mathrm{p}$ \\
\hline \multicolumn{4}{|l|}{ Sexo } \\
\hline Masculino & 1,00 & & \\
\hline Feminino & 2,52 & $1,90-3,34$ & $<0,001$ \\
\hline \multicolumn{4}{|l|}{ Escolaridade } \\
\hline Analfabetos & 2,12 & $1,06-4,22$ & 0,033 \\
\hline 1-4 anos do Ensino Fundamental incompleto & 2,05 & $1,19-3,53$ & 0,010 \\
\hline 5-8 anos do Ensino Fundamental completo & 1,84 & $0,99-3,39$ & 0,510 \\
\hline Ensino Médio incompleto & 1,07 & $0,53-2,15$ & 0,846 \\
\hline Ensino Médio completo & 1,00 & & \\
\hline \multicolumn{4}{|l|}{ Ocupação } \\
\hline Trabalhadores em atividade & 1,00 & & \\
\hline Estudantes & 0,71 & $0,46-1,09$ & 0,118 \\
\hline Desempregados & 2,40 & $1,65-3,49$ & $<0,001$ \\
\hline Aposentados por invalidez/em benefício-saúde & 2,62 & $1,71-4,00$ & $<0,001$ \\
\hline Aposentados por tempo de serviço & 0,86 & $0,35-2,09$ & 0,739 \\
\hline Donas-de-casa & 1,57 & $1,02-2,43$ & 0,041 \\
\hline
\end{tabular}


população de baixo nível sócio-econômico; as mesmas variáveis demográficas foram estudadas, com exceção de situação ocupacional e verificação da associação por meio de análise multivariada. Por isso tomamos como comparação. Participaram do estudo 2.337 indivíduos maiores de 14 anos, sendo 1.211 pertencentes às áreas de PSF. Utilizou-se como ponto de corte para considerar caso suspeito de THAS o proposto por Mari \&Williams 2 na década de 1980, ou seja, 7/8 para mulheres e $5 / 6$ para homens. A prevalência encontrada foi de 24,95\% (IC95\%: 22,04-28,1), sendo que para as duas áreas de PSF foi de $24,1 \%$ (IC95\%: 20,1-28,6) 6. Comparando a prevalência de THAS obtida no estudo conduzido em São Paulo com o presente estudo, podemos verificar que na população estudada em Santa Cruz do Sul houve uma prevalência mais alta.

Um outro estudo foi realizado em Pelotas, Rio Grande do Sul, Brasil, com 1.800 indivíduos representativos da população urbana daquela cidade. O ponto de corte considerado foi também de 7/8 para mulheres e de 5/6 para homens. A estimativa de THAS foi de 28,5\% (IC95\%: 26,4-30,6) 4, também abaixo da prevalência encontrada em Santa Cruz do Sul. Os outros dois estudos foram realizados no Estado de Pernambuco, Brasil, sendo um em parte da área urbana de Olinda 5 e outro na Zona da Mata Pernambucana 3. No primeiro a prevalência encontrada foi de $35 \%$, e no segundo, de $36 \%$ (não fornecem intervalos de confiança). Entretanto, o ponto de corte utilizado para considerar caso suspeito pelo SRQ-20 nesses dois estudos foi de $5 / 6$, ou seja, dois pontos abaixo do ponto de corte considerado no presente estudo (7/8).

Dentre os artigos de outros países identificados, encontramos prevalências variando de 11,7\% em Addis Ababa, capital da Etiópia 17, a $28 \%$ na comunidade urbana de Dhaka, em Bangladesh 18. A constatação de prevalência mais alta de THAS em nosso estudo é relevante, visto que o município onde o estudo foi realizado tem apresentado nos últimos anos alta incidência de mortalidade por suicídio (que se mantém em torno de 4,5 vezes maior que a média nacional: 4,5/100 mil vs. 21/100 mil para o ano de 2001) 19, embora deva ser feita a ressalva de que estes resultados referem-se a apenas uma área do referido município.

Em relação às associações de presença de THAS com variáveis demográficas, na análise univariada encontramos associação com sexo, idade, situação cojugal, escolaridade e situação ocupacional. Destas, duas variáveis perderam significância na associação com transtorno mental não psicótico na análise multivariada. São elas idade e situação conjugal.
Nossos achados em relação à associação positiva de sexo feminino com THAS estão de acordo com os relatados pela literatura desde as primeiras investigações epidemiológicas de base populacional na área de saúde mental 20,21, assim como estão de acordo com os quatro estudos brasileiros utilizando SRQ-20 citados acima $3,4,5,6$. As possíveis causas da associação com sexo feminino estão por ser esclarecidas, mas parece haver uma predisposição sócio-biológica para isto, estando o sistema neuroendocrinológico e o papel social interagindo de forma a aumentar a suscetibilidade 22,23.

Em relação à idade, alguns estudos têm demonstrado uma correlação linear com diagnóstico psiquiátrico. Entre as possíveis causas estão maior ocorrência de eventos de vida precipitantes de sintomas, maior tempo para manifestação de patologias, acúmulo de doenças crônicas, dificuldades econômicas e isolamento social 22 . Apesar disso, neste estudo o aumento de presença de THAS em função da idade não se manteve quando ajustado para as demais variáveis analisadas. No estudo de São Paulo a variável idade manteve-se associada com THAS após análise multivariada apenas na categoria 45 a 54 anos de idade 6 .

A associação entre situação conjugal e THAS é assunto controverso na literatura. Enquanto alguns estudos encontram associação, outros não. No estudo de São Paulo, por exemplo, casados apresentaram menores prevalências de THAS, mesmo após ajustados para outras variáveis, que solteiros, divorciados, viúvos e "concubinato" 6 . Na presente pesquisa houve significância na associação entre situação conjugal e THAS, com OR significativamente maiores entre os divorciados quando comparados com casados, na análise univariada. Porém, essa significância desaparece na análise multivariada. Ou seja, a associação entre situação conjugal e THAS não se deveu à variável propriamente dita, e sim ao fato de ser intermediada pelas variáveis: sexo, escolaridade e ocupação. Possivelmente a controvérsia em relação à associação da variável situação conjugal com THAS possa ser melhor esclarecida em estudos futuros, controlando a interferência de outras variáveis. $\mathrm{O}$ mesmo sugere-se em relação à idade.

Desempregados, aposentados por invalidez ou em benefício por problemas de saúde e donas-de-casa apresentaram chances significativamente maiores de apresentar THAS que os trabalhadores em atividade. Pode-se verificar que, após ajuste para outras variáveis demográficas, os indivíduos em situação ocupacional inativa por falta de emprego ou por problemas de saúde mantiveram a associação significativa. O estu- 
do de São Paulo não incluiu essa variável. Esses achados estão de acordo com a literatura, especialmente em relação à associação com desemprego, que é classicamente relatada em outros estudos 1,3,5. Entretanto, devemos ressaltar que de uma forma geral o trabalhador em atividade será mais sadio que os desempregados e, mais ainda, que os afastados do trabalho por problemas de saúde 8 .

Baixa escolaridade tem sido associada à presença de THAS. No estudo de São Paulo, escolaridade esteve inversamente associada a THAS; quando tomado por referência à categoria ensino superior, houve associação inversa com todas as demais categorias exceto ensino médio incompleto. Esse padrão de associação mantevese mesmo após ajuste para outras variáveis ${ }^{6}$. No presente estudo, na variável escolaridade encontramos prevalências crescentes deTHAS partindo da categoria ensino médio completo até analfabetos. Como resultado, obtivemos OR ajustadas e não ajustadas significativamente maiores entre indivíduos com Ensino Fundamental (completo e incompleto) e analfabetos quando comparados aos com ensino médio completo.

Este estudo não permite determinação de causalidade nas associações observadas, visto que é um estudo transversal. Entretanto, algumas considerações serão feitas. No campo da psiquiatria social, as teorias dos eventos de vida produtores de estresse, teoria dos papéis sociais e do suporte social, têm sido há anos cogitadas como explicativas para a associação de sexo feminino e indicadores de condições sócio-econômicas desfavoráveis com THAS. A primeira refere-se ao impacto sócio-biológico dos eventos estressores nos indivíduos aumentando suas chances de manifestarem sintomas de THAS; a segunda propõe que situações de desequilíbrio entre dominação e subordinação econômica, política e social agiriam como precipitantes de THAS 3,24; e a última afirma que o suporte social age como fator protetor para THAS, pois minimizaria os efeitos dos estressores de vida e significaria relacionamentos provedores de apoio promovendo bem-estar 24 .

No entanto não se pode descartar uma causalidade reversa, ou seja, que THAS sejam os precipitantes, e não a conseqüência de pior performance social, acadêmica e laboral. Essas considerações ganham força quando as evidências se acumulam no sentido de demonstrar que esses transtornos se manifestam precocemente na vida, e muitas vezes iniciam com longos períodos de prodomos e de sintomas subclínicos 25. Dessa forma permanecem sem diagnóstico e sem intervenções terapêuticas por muito tempo, causando prejuízos precoces em termos de evolução educacional e de preparação para o mercado de trabalho, o que em última análise culminaria em situação ocupacional desfavorável e indicadores de situação sócio-econômica também desfavoráveis. Além disso, não podemos desprezar a questão genética desses transtornos, que podem afetar os portadores de duas formas: através do modelo diátese-estresse, em que os estressores na vida produzirão sintomas nos indivíduos biológica e geneticamente suscetíveis, com maior ênfase nas questões biológicas que ambientais; e por meio de um contexto familiar comprometido desde muito cedo na vida, culminando em oportunidades mais limitadas de uma melhor situação sócio-econômica.

Considerando as evidências acima de alta prevalência de THAS na população, especialmente na que está em maior vulnerabilidade sócio-econômica, é oportuno recapitular que o último relatório da OMS sobre saúde mental propõe que o eixo do atendimento nesta área seja direcionado para centros de atendimento primário. O propósito é atuar em uma maior parcela da população, incluindo principalmente as mais vulneráveis 1,25 .

No Brasil isso indubitavelmente implica a inclusão desse tema nas ações do PSF, que tem se consolidado como estratégia de reorganização do sistema de atenção à saúde em todo país. Baseado na intervenção precoce, prevenção e educação em saúde, trabalha utilizando uma abordagem multidisciplinar e integrada à comunidade que atende. Tem apresentado elevada resolutividade no Brasil por meio da busca ativa de casos, trabalho em equipe e facilitação do acesso da população aos serviços de saúde 6 . Dessa forma, torna-se importante que as equipes de PSF estejam atentas aos aspectos da saúde mental da população atendida, a fim de que efetivamente apliquem a abordagem biopsicossocial a que se propõem, o que em última análise seria concretizado mais facilmente pela inclusão do tema saúde mental nas suas metas e prioridades em nível nacional.

Como sugestões para estudos futuros propomos a replicação deste tipo de estudo no Brasil, a realização de estudos longitudinais de base populacional para averiguar melhor as associações entre diagnóstico psiquiátrico e indicadores de desvantagem social, pesquisas de avaliação da lacuna existente no país entre demanda e oferta de assistência em saúde mental e trabalhos de avaliação da efetividade de oferta de tratamento para THAS em larga escala.

Entre as limitações mais importantes do estudo está o fato de a prevalência de THAS ter sido dada por um instrumento de rastreamento e não pelo padrão-ouro (entrevista psiquiátrica), embora tenhamos tentado atenuar esta limitação 
por meio de um estudo de validação prévio do SRQ-20, comparando-o com entrevista psiquiátrica utilizando o SCID-IV-TR 15 e estabelecer os pontos de corte para esta população ${ }^{16}$. Além disso, pode ter existido alguma influência dos indivíduos que se recusaram participar do estudo, tanto no sentido de aumentar quanto de diminuir a prevalência de THAS e causar algum tipo de interferência nas análises de associação.
Essa limitação tentou-se evitar convidando os moradores mais de uma vez para participar da pesquisa, sendo o convite feito pelo próprio agente comunitário de saúde, por intermédio do morador presente e por carta. Por fim, as conclusões deste estudo têm limitações quanto à sua validade para populações diferentes desta, especialmente, com maior diversidade nas variáveis demográficas.

\section{Resumo}

O objetivo principal deste estudo é estimar a prevalência de transtornos mentais de humor, ansiedade e somatização (THAS) utilizando o Self-Reporting Questionnaire-20 Itens (SRQ-20) em comunidade atendida pelo Programa Saúde da Família (PSF) em Santa Cruz do Sul, Rio Grande do Sul, Brasil. Objetivo secundário é verificar a associação de THAS com variáveis demográficas. Todos os moradores da área do PSF avaliada maiores de 14 anos foram convidados a participar. A amostra total analisada foi composta de 1.122 indivíduos. A prevalência encontrada de THAS foi de $38 \%$ (IC95\%: 35, 12-40,88). Sexo feminino, baixa escolaridade e situação ocupacional desfavorável mostraram associação independente com THAS. A alta prevalência de THAS na área estudada demonstra a importância destes transtornos em termos de saúde pública, e pode trazer alguma contribuição para o entendimento da alta incidência de mortalidade por suicídio verificada em Santa Cruz do Sul na última década (4,66 vezes maior que a incidência nacional). Incluir o tema saúde mental nas metas e prioridades do PSF no Brasil é muito importante.

Transtornos Mentais; Saúde Mental; Programa Saúde da Família

\section{Colaboradores}

D. M. Gonçalves participou da elaboração e delineamento do projeto, pesquisa de campo, análise de dados e redação do artigo. F. Kapczinski colaborou na supervisão e orientação de todas as etapas da pesquisa e da elaboração do artigo.

\section{Agradecimentos}

Ao Conselho Nacional de Desenvolvimento Científico e Tecnológico (CNPq, projeto $\mathrm{n}^{\mathrm{o}}$. 401868/2005-2) pelo financiamento. 


\section{Referências}

1. World Health Organization. The World Health Report 2001. Mental health new understanding, new hope. Geneva: World Health Organization; 2001.

2. Mari JJ, Williams P. A comparison of the validity of two psychiatric screening questionnaires (GHQ12 and SRQ-20) in Brazil, using relative operating characteristic (ROC) analysis. Psychol Med 1985; 15:651-9.

3. Costa AG, Ludermir AB. Transtornos mentais comuns e apoio social: estudo em comunidade rural da Zona da Mata de Pernambuco, Brasil. Cad Saúde Pública 2005; 21:73-9.

4. Costa JSD, Menezes AMB, Olinto MTA, Gigante DP, Macedo S, Britto MAP. Prevalência de distúrbios psiquiátricos menores na cidade de Pelotas, RS. Rev Bras Epidemiol 2002; 5:164-73.

5. Ludermir AB, Melo Filho DA. Condições de vida e estrutura ocupacional associadas a transtornos mentais comuns. Rev Saúde Pública 2002; 36:213-21.

6. Maragno L, Goldbaum M, Gianini RJ, Novaes HMD, César CLG. Prevalência de transtornos mentais comuns em populações atendidas pelo Programa Saúde da Família (QUALIS) no Município de São Paulo, Brasil. Cad Saúde Pública 2006; 22:1639-48.

7. Goldberg DP, Huxley P. Common mental disorders: a bio-social model. London: Tavistock; 1992.

8. Pereira MG. Indicadores de saúde. In: Pereira MG, organizador. Epidemiologia: teoria e prática. $5 \underline{\text { a }} \mathrm{Ed}$. Rio de Janeiro: Editora Guanabara Koogan; 2001. p. 49-73.

9. Nedel FB, Rocha M, Pereira J. Anos de vida perdidos por mortalidade: um dos componentes da carga de doenças Rev Saúde Pública 1999; 33:461-9.

10. Harding TW, Arango MV, Baltazar J, Climent CE, Ibrahim $\mathrm{HH}$, Ladrido-Ignacio L, et al. Mental disorders in primary health care: a study of their frequency and diagnosis in four developing countries. Psychol Med 1980; 10:231-41

11. World Health Organization. A user's guide to the Self-Reporting Questionnaire. Geneva:World Health Organization; 1993.

12. Goldberg DP. The detection of psychiatric illness by questionnaire. London: Oxford University Press; 1972. (Maudsley Monograph $n^{\circ} .21$ ).

13. American Psychiatric Association. Diagnostic and statistical manual of mental disorders. $3^{\text {rd }}$ Ed. Washington DC: American Psychiatric Association; 1980.
14. Biometrics Research Department, Columbia University. Structured clinical interview for DSM disorders (SCID). http://cpmcnet.columbia.edu/dept/ scid/ (acessado em 08/Set/2007).

15. American Psychiatric Association. DSM-IV-TR: manual diagnóstico e estatístico de transtornos mentais. 4a Ed. Porto Alegre: Editora Artmed; 2002.

16. Gonçalves DM, Stein AT, Kapczinski F. Avaliação de desempenho do Self-Reporting Questionnaire como instrumento de rastreamento psiquiátrico: um estudo comparativo com o Structured Clinical Interview for DSM-IV-TR. Cad Saúde Pública 2008; 24:380-90.

17. Kebede D, Alem A, Rashid E. The prevalence and socio-demographic correlates of mental distress in Addis Ababa, Ethiopia. Acta Psychiatr Scand Suppl 1999; 397:5-10.

18. Islam MM, Ali M, Ferroni P, Underwood P, Alam MF. Prevalence of psychiatric disorders in an urban community in Bangladesh. Gen Hosp Psychiatry 2003; 25:353-7.

19. Secretaria de Vigilância em Saúde, Ministério da Saúde. Perfil epidemiológico dos suicídios: Brasil e regiões, 1996-2002. Brasília: Secretaria de Vigilância em Saúde, Ministério da Saúde; 2005.

20. Finlay-Jones RA, Burvill PW. The prevalence of minor psychiatric morbidity in the community. Psychol Med 1977; 7:475-89.

21. Brenner SO, Levi L. Long-term unemployment among women in Sweden. Soc Sci Med 1987; 25:153-61.

22. Linzer M, Spitzer R, Kroenke K, Williams JB, Hahn S, Brody D, et al. Gender, quality of life and mental disorders in primary care: results from the PRIMEMD 1000 study. Am J Med 1996; 101:526-33.

23. Gove WR, Tudor JF. Adult sex roles and mental illness. Am J Sociol 1973; 78:812-35.

24. Sherman AM. Social relations and depressive symptoms in older adults with knee osteoarthritis. Soc Sci Med 2003; 56:247-57.

25. Cross-national comparisons of the prevalences and correlates of mental disorders. WHO International Consortium in Psychiatric Epidemiology. Bull World Health Organ 2000; 78:413-26.

Recebido em 13/Set/2007

Versão final reapresentada em 19/Nov/2007 Aprovado em 26/Dez/2007 